Communications in Physics, Vol. 24, No. 2 (2014), pp. 97-112

\title{
NEUTRAL CURRENT IN REDUCED MINIMAL 3-3-1 MODEL
}

\author{
VU THI NGOC HUYEN AND HOANG NGOC LONG \\ Institute of Physics, Vietnam Academy of Science and Technology \\ TRAN THANH LAM \\ Department of Physics, Kien Giang Community College \\ VO QUOC PHONG \\ Department of Physics, Ho Chi Minh city University of Natural Science, Vietnam
}

E-mail: hnlong@iop.vast.ac.vn

Received 24 February 2014

Accepted for publication 04 April 2014

\begin{abstract}
This work is devoted for gauge boson sector of the recently proposed model based on $\mathrm{SU}(3)_{C} \otimes \mathrm{SU}(3)_{L} \otimes$ $\mathrm{U}(1)_{X}$ group with minimal content of leptons and Higgses. The limits on the masses of the bilepton gauge bosons and on the mixing angle among the neutral ones are deduced. Using the Fritzsch anzats on quark mixing, we show that the third family of quarks should be different from the first two. We obtain a lower bound on mass of the new heavy neutral gauge boson as $4.032 \mathrm{TeV}$. Using data on branching decay rates of the $Z$ boson, we can fix the limit to the $Z$ and $Z^{\prime}$ mixing angle $\phi$ as $-0.001 \leq \phi \leq 0.0003$.
\end{abstract}

Keywords: extended standard model.

\section{INTRODUCTION}

The experimental evidences of nonzero neutrino masses and mixing [1] have shown that the standard model (SM) of fundamental particles and interactions must be extended. Among many extensions of the SM known today, the models based on gauge symmetry $\mathrm{SU}(3)_{C} \otimes \mathrm{SU}(3)_{L} \otimes$ $\mathrm{U}(1)_{X}$ (so-called 3-3-1 models) [2,3] has interesting features. First, $\left[\mathrm{SU}(3)_{L}\right]^{3}$ anomaly cancellation requires that the number of $\mathrm{SU}(3)_{L}$ fermion triplets must equal to that of antitriplets. If these multiplets are respectively enlarged from those of the SM, the fermion family number is deduced to be a multiple of the fundamental color number, which is three, coinciding with the observation. In addition, one family of quarks has to transform under $\mathrm{SU}(3)_{L}$ differently from the other two. This can lead to an explanation why the top quark is uncharacteristically heavy.

One of the weaknesses of the mentioned 3-3-1 models that reduces their predictive possibility is a plenty or complication in the scalar sectors. The attempt on this direction to realize simpler scalar sectors has been recently constructed 3-3-1 model with minimal Higgs sector called the economical 3-3-1 model [4,5]. The 3-3-1 model with minimal content of fermions and Higgs sector (called the reduced minimal (RM) 3-3-1 model) has also been constructed in [6]. 
The aim of this work is to present in details the recently proposed model with focus on gauge boson sector, and to correct some misprints in the original work [6]. The article is organized as follows: In section II, in order to make his work self-contained, we review the basics of the reduced minimal 3-3-1 model. Section III is devoted for the Higgs sector. In section IV, we give more details on gauge bosons: their masses and mixing. Fermion masses and Yukawa interactions (with some corrections) are given in section V. The charged and neutral currents are presented in section VI, and using the obtained results we get the constraints on masses of the new neutral $Z^{\prime}$ gauge boson in section VII. Section VIII is devoted for the $Z$ decay, from which the limit on $Z-Z^{\prime}$ mixing angle $\phi$ is derived. In the last section, we summarize our main results.

\section{PARTICLE CONTENT}

The fermion content of the model under consideration is the same as in the minimal 3-3-1 model [2,6]. The left-handed leptons and quarks transform under the $S U(3)_{L}$ gauge group as the triplets

$$
\begin{aligned}
f_{a L} & =\left(\begin{array}{c}
\nu_{\ell_{a}} \\
\ell_{a} \\
\ell_{a}^{c}
\end{array}\right)_{L} \sim(\mathbf{3}, 0) \quad Q_{1 L}=\left(\begin{array}{c}
u_{1} \\
d_{1} \\
T
\end{array}\right)_{L} \sim\left(\mathbf{3}, \frac{2}{3}\right), \\
Q_{i L} & =\left(\begin{array}{c}
d_{i} \\
-u_{i} \\
D_{i}
\end{array}\right)_{L} \sim\left(\mathbf{3}^{*},-\frac{1}{3}\right)
\end{aligned}
$$

where $a=1,2,3$ and $i=2,3$. The $T$ exotic quark carries $5 / 3$ units of positron's electric charge, while $D_{2}$ and $D_{3}$ carry $-4 / 3$ each one. In Eqs. (1) the numbers $0,2 / 3$, and $-1 / 3$ are the $\mathrm{U}(1)_{X}$ charges. The right-handed quarks are singlets of the $\mathrm{SU}(3)_{L}$ group,

$$
\begin{array}{ll}
u_{R}^{a} \sim(\mathbf{1}, 2 / 3), & d_{R}^{a} \sim(\mathbf{1},-1 / 3), a=1,2,3, \\
T_{R} \sim(\mathbf{1}, 5 / 3), & D_{i R} \sim(\mathbf{1},-4 / 3) .
\end{array}
$$

The charge operator is defined by

$$
\frac{Q}{e}=\frac{\lambda_{3}}{2}-\frac{\sqrt{3}}{2} \lambda_{8}+X
$$

where $\lambda_{3}$ and $\lambda_{8}$ are the diagonal Gell-Mann matrices. Note that for antitriplet, we have to replace the Gell-mann matrix by $\bar{\lambda}=-\lambda^{*}$.

The scalar sector contains only two Higgs scalar triplets [6]

$$
\rho=\left(\begin{array}{c}
\rho^{+} \\
\rho^{0} \\
\rho^{++}
\end{array}\right) \sim(\mathbf{3}, 1), \quad \chi=\left(\begin{array}{c}
\chi^{-} \\
\chi^{--} \\
\chi^{0}
\end{array}\right) \sim(\mathbf{3},-1) .
$$

This minimal content of Higgs sector is enough to break the symmetry spontaneously and generate the masses of fermions and gauge bosons in the model [6]. The neutral scalar fields develop the vacuum expectation values (VEVs) $\left\langle\rho^{0}\right\rangle=\frac{v_{\rho}}{\sqrt{2}}$ and $\left\langle\chi^{0}\right\rangle=\frac{v_{\chi}}{\sqrt{2}}$, with $v_{\rho}=246 \mathrm{GeV}$.

The pattern of symmetry breaking is

$$
\mathrm{SU}(3)_{L} \otimes \mathrm{U}(1)_{X} \stackrel{\left\langle\chi^{0}\right\rangle}{\longrightarrow} \mathrm{SU}(2)_{L} \otimes \mathrm{U}(1)_{Y} \stackrel{\left\langle\rho^{0}\right\rangle}{\longrightarrow} \mathrm{U}(1)_{\mathrm{em}}
$$


and so, we can expect

$$
v_{\chi} \gg v_{\rho}
$$

Since lepton and antilepton were put in the same triplet, therefore in the model under consideration, lepton number is not conserved. It is better to work with a new conserved charge $\mathcal{L}$ commuting with the gauge symmetry $[7,8]$ and related to the ordinary lepton number by diagonal matrices $L=\frac{4}{\sqrt{3}} T_{8}+\mathcal{L}$.

Another useful conserved charge $B$ is usual baryon number [8] $B=\mathcal{B} I$. These numbers are given $[7,8]$ in the Table 1

Table 1. $\mathcal{B}$ and $\mathcal{L}$ charges for multiplets in the RM 331 model.

\begin{tabular}{|l|ccccccccc|}
\hline Multiplet & $\chi$ & $\rho$ & $Q_{1 L}$ & $Q_{i L}$ & $u_{a R}$ & $d_{a R}$ & $T_{R}$ & $D_{i R}$ & $f_{a L}$ \\
\hline $\mathcal{B}$ charge & 0 & 0 & $\frac{1}{3}$ & $\frac{1}{3}$ & $\frac{1}{3}$ & $\frac{1}{3}$ & $\frac{1}{3}$ & $\frac{1}{3}$ & 0 \\
\hline $\mathcal{L}$ charge & $\frac{4}{3}$ & $-\frac{2}{3}$ & $-\frac{2}{3}$ & $\frac{2}{3}$ & 0 & 0 & -2 & 2 & $\frac{1}{3}$ \\
\hline
\end{tabular}

In Table 2, we list particles with non-zero lepton number.

Table 2. Nonzero lepton number $L$ of fields in the RM 331 model.

\begin{tabular}{|c|cc|ccc|cccc|}
\hline Fields & $l_{L}^{c}$ & $l_{L}$ & $\rho_{3}^{++}$ & $\chi_{1}^{-}$ & $\chi_{2}^{--}$ & $D_{i L}$ & $D_{i R}$ & $T_{L}$ & $T_{R}$ \\
\hline$L$ & -1 & 1 & -2 & 2 & 2 & 2 & 2 & -2 & -2 \\
\hline
\end{tabular}

Table 2 shows that the exotic quarks carry lepton number two. Hence they are bilepton quarks.

\section{HIGGS POTENTIAL}

The most general renormalizable scalar potential is given by [6]

$$
\begin{aligned}
V(\chi, \rho)= & \mu_{1}^{2} \rho^{\dagger} \rho+\mu_{2}^{2} \chi^{\dagger} \chi+\lambda_{1}\left(\rho^{\dagger} \rho\right)^{2}+\lambda_{2}\left(\chi^{\dagger} \chi\right)^{2} \\
& +\lambda_{3}\left(\rho^{\dagger} \rho\right)\left(\chi^{\dagger} \chi\right)+\lambda_{4}\left(\rho^{\dagger} \chi\right)\left(\chi^{\dagger} \rho\right),
\end{aligned}
$$

This potential is the simplest one since the number of free parameters is reduced from, at least, thirteen to only six.

Expansion of $\rho^{0}$ and $\chi^{0}$ around their VEVs is usually

$$
\rho^{0}, \chi^{0} \rightarrow \frac{1}{\sqrt{2}}\left(v_{\rho, \chi}+R_{\rho, \chi}+i I_{\rho, \chi}\right) .
$$

Substituting the expansion in (6) into the above potential we obtain the following set of minimum constraint equations [6]

$$
\begin{aligned}
& \mu_{1}^{2}+\lambda_{1} v_{\rho}^{2}+\frac{\lambda_{3} v_{\chi}^{2}}{2}=0 \\
& \mu_{2}^{2}+\lambda_{2} v_{\chi}^{2}+\frac{\lambda_{3} v_{\rho}^{2}}{2}=0 .
\end{aligned}
$$

This potential immediately gives us two charged Goldstones bosons $\rho^{ \pm}$and $\chi^{ \pm}$which are eaten by the gauge bosons $W^{ \pm}$and $V^{ \pm}$. 
In the doubly charged scalars, the mass matrix in the basis $\left(\chi^{++}, \rho^{++}\right)$is given by

$$
\frac{\lambda_{4}}{2}\left(\begin{array}{cc}
v_{\rho}^{2} & v_{\chi} v_{\rho} \\
v_{\chi} v_{\rho} & v_{\chi}^{2}
\end{array}\right)
$$

This matrix has the following squared mass eigenvalues

$$
m_{\tilde{h}^{--}}^{2}=0 \quad \text { and } \quad m_{h^{--}}^{2}=\frac{\lambda_{4}}{2}\left(v_{\chi}^{2}+v_{\rho}^{2}\right)
$$

where the corresponding eigenstates are

$$
\left(\begin{array}{c}
\tilde{h}^{++} \\
h^{++}
\end{array}\right)=\left(\begin{array}{cc}
c_{\alpha} & -s_{\alpha} \\
s_{\alpha} & c_{\alpha}
\end{array}\right)\left(\begin{array}{c}
\chi^{++} \\
\rho^{++}
\end{array}\right)
$$

with

$$
c_{\alpha}=\frac{v_{\chi}}{\sqrt{v_{\chi}^{2}+v_{\rho}^{2}}}, s_{\alpha}=\frac{v_{\rho}}{\sqrt{v_{\chi}^{2}+v_{\rho}^{2}}} .
$$

In the neutral scalar sector, in the basis $\left(R_{\chi}, R_{\rho}\right)$, the mass matrix takes the following form

$$
\left(\begin{array}{cc}
\lambda_{2} v_{\chi}^{2} & \frac{1}{2} \lambda_{3} v_{\chi} v_{\rho} \\
\frac{1}{2} \lambda_{3} v_{\chi} v_{\rho} & \lambda_{1} v_{\rho}^{2}
\end{array}\right)
$$

This matrix gives us two eigenvalues

$$
\begin{aligned}
& m_{h_{1}}^{2}=\frac{1}{2} v_{\chi}^{2}\left(\lambda_{1} t^{2}+\lambda_{2}-\sqrt{\Delta}\right), \\
& m_{h_{2}}^{2}=\frac{1}{2} v_{\chi}^{2}\left(\lambda_{1} t^{2}+\lambda_{2}+\sqrt{\Delta}\right),
\end{aligned}
$$

where $t \equiv \frac{v_{\rho}}{v_{\chi}}$ and

$$
\Delta=\left(\lambda_{1} t^{2}-\lambda_{2}\right)^{2}+\lambda_{3}^{2} t^{2}
$$

The corresponding eigenvectors are

$$
\left(\begin{array}{c}
h_{1} \\
h_{2}
\end{array}\right)=\left(\begin{array}{cc}
-s_{\beta} & c_{\beta} \\
c_{\beta} & s_{\beta}
\end{array}\right)\left(\begin{array}{c}
R_{\chi} \\
R_{\rho}
\end{array}\right)
$$

with

$$
c_{\beta}=\frac{1}{\sqrt{2}}\left(1-\frac{\lambda_{1} t^{2}-\lambda_{2}}{\sqrt{\Delta}}\right)^{\frac{1}{2}}, \quad s_{\beta}=\frac{1}{\sqrt{2}}\left(1+\frac{\lambda_{1} t^{2}-\lambda_{2}}{\sqrt{\Delta}}\right)^{\frac{1}{2}} .
$$

In the neutral pseudoscalar sector, there are two Goldstones bosons $I_{\rho}$ and $I_{\chi}$ which are eaten by the neutral gauge bosons $Z$ and $Z^{\prime}$, respectively.

In the effective limit: $v_{\chi} \gg v_{\rho}$ we have

$$
\begin{aligned}
& c_{\alpha} \approx 1, s_{\alpha} \approx 0, \quad \sqrt{\Delta} \approx \lambda_{2}-\lambda_{1} t^{2}+\frac{\lambda_{3}^{2}}{2 \lambda_{2}} t^{2}, \\
& c_{\beta} \approx 1-\frac{\lambda_{3}^{2}}{8 \lambda_{2}^{2}} t^{2}, \quad s_{\beta} \approx \frac{\lambda_{3} t}{2 \lambda_{2}}
\end{aligned}
$$

This gives the following consequences:

(1) The Goldstones boson $\tilde{h}^{--} \approx \chi^{--}$and one physical doubly charged Higgs boson is $h^{++} \approx \rho^{++}$. 
(2) Masses of neutral Higgs bosons

$$
m_{h_{1}}^{2}=\left(\lambda_{1}-\frac{\lambda_{3}^{2}}{4 \lambda_{2}}\right) v_{\rho}^{2}, \quad m_{h_{2}}^{2}=\lambda_{2} v_{\chi}^{2}+\frac{\lambda_{3}^{2}}{4 \lambda_{2}} v_{\rho}^{2},
$$

(3) The positiveness of masses yields: $\lambda_{1}>0, \lambda_{2}>0,4 \lambda_{1} \lambda_{2}>\lambda_{3}^{2}$.

Let us resume content of the Higgs sector: the physical scalar spectrum of the RM331 model is composed by a doubly charged scalar $h^{++}$and two neutral scalars $h_{1}$ and $h_{2}$. Since the lightest neutral field, $h_{1}$, is basically a $S U(2)_{L}$ component in the linear combination as in Eq. (13), we identify it as the standard Higgs boson. Thus

$$
\rho=\left(\begin{array}{c}
G_{W^{+}} \\
\frac{v_{\rho}}{\sqrt{2}}+\frac{1}{\sqrt{2}}\left(h_{1}+i G_{Z}\right) \\
h^{++}
\end{array}\right), \quad \chi=\left(\begin{array}{c}
G_{V^{-}} \\
G_{U^{--}} \\
\frac{v_{\chi}}{\sqrt{2}}+\frac{1}{\sqrt{2}}\left(h_{2}+i G_{Z^{\prime}}\right)
\end{array}\right)
$$

Note that $h^{--}$carries lepton number two. Hence, it is scalar bilepton.

\section{GAUGE BOSONS}

The masses of gauge bosons appear in the Lagrangian part

$$
\mathcal{L}=\left(D_{\mu} \chi\right)^{\dagger}\left(D^{\mu} \chi\right)+\left(D_{\mu} \rho\right)^{\dagger}\left(D^{\mu} \rho\right)
$$

where

$$
D_{\mu}=\partial_{\mu}-i g A_{\mu}^{a} \frac{\lambda^{a}}{2}-i g_{X} X \frac{\lambda_{9}}{2} B_{\mu},
$$

with $\lambda_{9}=\sqrt{\frac{2}{3}} \operatorname{diag}(1,1,1)$ so that $\operatorname{Tr}\left(\lambda_{9} \lambda_{9}\right)=2$. The coupling constants of $S U(3)_{L}$ and $U(1)_{X}$ satisfy the following relation

$$
\frac{g_{X}^{2}}{g^{2}}=\frac{6 s_{W}^{2}}{1-4 s_{W}^{2}}
$$

where we have used the notations $c_{W}=\cos \theta_{W}, s_{W}=\sin \theta_{W}, t_{W}=\tan \theta_{W}$ with $\theta_{W}$ being the Weinberg mixing angle. Substitution of the expansion in the Eq. (6) into (16) leads to the following result: The eigenstates of the charged gauge bosons and their respective masses are given by

$$
\begin{aligned}
& W^{ \pm}=\frac{A^{1} \mp i A^{2}}{\sqrt{2}} \rightarrow M_{W^{ \pm}}^{2}=\frac{g^{2} v_{\rho}^{2}}{4}, \\
& V^{ \pm}=\frac{A^{4} \pm i A^{5}}{\sqrt{2}} \rightarrow M_{V^{ \pm}}^{2}=\frac{g^{2} v_{\chi}^{2}}{4}, \\
& U^{ \pm \pm}=\frac{A^{6} \pm i A^{7}}{\sqrt{2}} \rightarrow M_{U^{ \pm \pm}}^{2}=\frac{g^{2}\left(v_{\rho}^{2}+v_{\chi}^{2}\right)}{4}
\end{aligned}
$$

From (19), it follows that $v_{\rho}=246 \mathrm{GeV}$. Note that there is mass splitting of the charged gauge bosons

$$
M_{U}^{2}-M_{V}^{2}=M_{W}^{2}
$$


The covariant derivative of the lepton triplets is

$$
\frac{g}{2} \vec{\lambda} \vec{A}_{\mu}=\left(\begin{array}{lcr}
\frac{g}{2}\left(A_{\mu}^{3}+\frac{1}{\sqrt{3}} A_{\mu}^{8}\right) & \frac{g}{\sqrt{2}} W_{\mu}^{+} & \frac{g}{\sqrt{2}} V_{\mu}^{-} \\
\frac{g}{\sqrt{2}} W_{\mu}^{-} & \frac{g}{2}\left(-A_{\mu}^{3}+\frac{1}{\sqrt{3}} A_{\mu}^{8}\right) & \frac{g}{\sqrt{2}} U_{\mu}^{--} \\
\frac{g}{\sqrt{2}} V_{\mu}^{+} & \frac{g}{\sqrt{2}} U_{\mu}^{++} & -g \frac{1}{\sqrt{3}} A_{\mu}^{8}
\end{array}\right) .
$$

while for the anti-triplets we have

$$
\frac{g}{2} \vec{\lambda} \vec{A}_{\mu}=\left(\begin{array}{ccr}
-\frac{g}{2}\left(A_{\mu}^{3}+\frac{1}{\sqrt{3}} A_{\mu}^{8}\right) & -\frac{g}{\sqrt{2}} W_{\mu}^{-} & -\frac{g}{\sqrt{2}} V_{\mu}^{+} \\
-\frac{g}{\sqrt{2}} W_{\mu}^{+} & -\frac{g}{2}\left(-A_{\mu}^{3}+\frac{1}{\sqrt{3}} A_{\mu}^{8}\right) & -\frac{g}{\sqrt{2}} U_{\mu}^{++} \\
-\frac{g}{\sqrt{2}} V_{\mu}^{-} & -\frac{g}{\sqrt{2}} U_{\mu}^{--} & g \frac{1}{\sqrt{3}} A_{\mu}^{8}
\end{array}\right),
$$

where $\bar{\lambda}=-\lambda^{*}$.

In the neutral gauge boson sector, with the basis $\left(A_{\mu}^{3}, A_{\mu}^{8}, B_{\mu}\right)$, mass matrix is given by

$$
M^{2}=\frac{g^{2}}{4}\left(\begin{array}{ccc}
v_{\rho}^{2} & -\frac{v_{\rho}^{2}}{\sqrt{3}} & -2 \kappa v_{\rho}^{2} \\
-\frac{v_{\rho}^{2}}{\sqrt{3}} & \frac{1}{3}\left(v_{\rho}^{2}+4 v_{\chi}^{2}\right) & \frac{2}{\sqrt{3}}\left(v_{\rho}^{2}+2 v_{\chi}^{2}\right) \\
-2 \kappa v_{\rho}^{2} & \frac{2}{\sqrt{3}}\left(v_{\rho}^{2}+2 v_{\chi}^{2}\right) & 4 \kappa^{2}\left(v_{\rho}^{2}+v_{\chi}^{2}\right)
\end{array}\right)
$$

where $\kappa=\frac{g_{X}}{g}$. We can easily identify the photon field $A_{\mu}$ as well as the massive bosons $Z$ and $Z^{\prime}[9]$

$$
\begin{aligned}
& A_{\mu}=s_{W} A_{\mu}^{3}+c_{W}\left(\sqrt{3} t_{W} A_{\mu}^{8}+\sqrt{1-3 t_{W}^{2}} B_{\mu}\right), \\
& Z_{\mu}=c_{W} A_{\mu}^{3}-s_{W}\left(\sqrt{3} t_{W} A_{\mu}^{8}+\sqrt{1-3 t_{W}^{2}} B_{\mu}\right),
\end{aligned}
$$

and

$$
Z_{\mu}^{\prime}=-\sqrt{1-3 t_{W}^{2}} A_{\mu}^{8}+\sqrt{3} t_{W} B_{\mu}
$$

where the mass-squared matrix for $\left\{Z, Z^{\prime}\right\}$ is given by

$$
\mathcal{M}^{2}=\left(\begin{array}{cc}
M_{Z}^{2} & M_{Z Z^{\prime}}^{2} \\
M_{Z Z^{\prime}}^{2} & M_{Z^{\prime}}^{2}
\end{array}\right)
$$

with

$$
\begin{aligned}
M_{Z}^{2} & =\frac{1}{4} \frac{g^{2}}{\cos ^{2} \theta_{W}} v_{\rho}^{2}, \\
M_{Z^{\prime}}^{2} & =\frac{1}{3} g^{2}\left[\frac{\cos ^{2} \theta_{W}}{1-4 \sin ^{2} \theta_{W}} v_{\chi}^{2}+\frac{1-4 \sin ^{2} \theta_{W}}{4 \cos ^{2} \theta_{W}} v_{\rho}^{2}\right], \\
M_{Z Z^{\prime}}^{2} & =\frac{1}{4 \sqrt{3}} g^{2} \frac{\sqrt{1-4 \sin ^{2} \theta_{W}}}{\cos ^{2} \theta_{W}} v_{\rho}^{2} .
\end{aligned}
$$

Diagonalizing the mass matrix gives the mass eigenstates $Z_{1}$ and $Z_{2}$ which can be taken as mixtures,

$$
\begin{aligned}
& Z_{1}=Z \cos \phi-Z^{\prime} \sin \phi, \\
& Z_{2}=Z \sin \phi+Z^{\prime} \cos \phi .
\end{aligned}
$$


The mixing angle $\phi$ is given by

$$
\tan 2 \phi=\frac{M_{Z}^{2}-M_{Z_{1}}^{2}}{M_{Z_{2}}^{2}-M_{Z}^{2}}
$$

where $M_{Z^{1}}$ and $M_{Z^{2}}$ are the physical mass eigenvalues

$$
\begin{aligned}
& M_{Z_{1}}^{2}=\frac{1}{2}\left\{M_{Z^{\prime}}^{2}+M_{Z}^{2}-\left[\left(M_{Z^{\prime}}^{2}-M_{Z}^{2}\right)^{2}-4\left(M_{Z Z^{\prime}}^{2}\right)^{2}\right]^{1 / 2}\right\}, \\
& M_{Z_{2}}^{2}=\frac{1}{2}\left\{M_{Z^{\prime}}^{2}+M_{Z}^{2}+\left[\left(M_{Z^{\prime}}^{2}-M_{Z}^{2}\right)^{2}-4\left(M_{Z Z^{\prime}}^{2}\right)^{2}\right]^{1 / 2}\right\} .
\end{aligned}
$$

From the symmetry breaking hierarchy, $v_{\chi} \gg v_{\rho}$, we obtain the lower mass bound of $Z_{2}$ [9]

$$
\begin{aligned}
M_{Z_{2}} & \geq \sqrt{\frac{4}{3}} \frac{\cos ^{2} \theta_{W}\left(M_{Z_{2}}\right)}{\sqrt{1-4 \sin ^{2} \theta_{W}\left(M_{Z_{2}}\right)}} M_{Z_{1}} \\
& \geq 400 \mathrm{GeV} .
\end{aligned}
$$

For practical calculations, it is useful the following relations

$$
\begin{aligned}
& A_{\mu}^{3}=c_{W} Z_{\mu}+s_{W} A_{\mu} \\
& A_{\mu}^{8}=\sqrt{3} t_{W} s_{W} Z_{\mu}+\sqrt{1-3 t_{W}^{2}} Z_{\mu}^{\prime}-\sqrt{3} s_{W} A_{\mu}, \\
& B_{\mu}=-s_{W} \sqrt{1-3 t_{W}^{2}} Z_{\mu}+\sqrt{3} t_{W} Z_{\mu}^{\prime}+c_{W} \sqrt{1-3 t_{W}^{2}} A_{\mu} .
\end{aligned}
$$

Trilinear and quartic interactions of the gauge bosons are the same as in Ref. [10] Using data on the wrong muon decay [1]

$$
\operatorname{Br}\left(\mu \rightarrow e+\nu_{e}+\tilde{\nu}_{\mu}\right)<1.2 \% \quad \text { at } \quad 90 \% \quad \mathrm{CL}
$$

we get a lower limit on singly charged bilepton gauge boson as follows (see, the last reference in [3])

$$
M_{V} \geq 230 \mathrm{GeV}
$$

This means that the model works in quite lower energy limit available for example such as the CERN LHC.

\section{FERMION MASSES}

As in the original minimal version, in this model, the singlet right-handed lepton does not exist. Thus, the fermion masses are due to effective operators. The appropriate sources of mass for each fermion in the model are: the Yukawa couplings give the exotic quark masses [6]

$$
\begin{aligned}
L_{Y u k}^{\text {exot }}= & \lambda_{11}^{T} \bar{Q}_{1 L} \chi T_{R}+\lambda_{i j}^{D} \bar{Q}_{i L} \chi^{*} D_{j R}+H . c . \\
= & \lambda_{11}^{T}\left(\bar{u}_{1 L} \chi^{-}+\bar{d}_{1 L} \chi^{--}+\bar{T}_{L} \chi^{0}\right) T_{R} \\
& \lambda_{i j}^{D}\left(\bar{d}_{i L} \chi^{+}-\bar{u}_{i L} \chi^{++}+\bar{D}_{i L} \chi^{0 *}\right) D_{j R}+\text { H.c. }
\end{aligned}
$$

When the $\chi$ field develops its VEV, these couplings lead to the mass matrix in the basis $\left(T, D_{2}, D_{3}\right)$,

$$
M_{J}=\frac{v_{\chi}}{\sqrt{2}}\left(\begin{array}{ccc}
\lambda_{11}^{T} & 0 & 0 \\
0 & \lambda_{22}^{D} & \lambda_{23}^{D} \\
0 & \lambda_{32}^{D} & \lambda_{33}^{D}
\end{array}\right)
$$


which, after diagonalization, leads to mass eigenvalues at $v_{\chi}$ around few $\mathrm{TeV}$ scale [6].

For the ordinary quarks, their masses come from both renormalizable Yukawa interactions and specific effective dimension-five operators given by

$$
\begin{aligned}
-L_{Y u k}^{u} & =\lambda_{i a}^{u} \bar{Q}_{i L} \rho^{*} u_{a R}+\frac{\lambda_{1 a}^{u}}{\Lambda} \varepsilon_{n m p}\left(\bar{Q}_{1 L n} \rho_{m}^{*} \chi_{p}^{*}\right) u_{a R}+H . c . \\
& =\lambda_{i a}^{u}\left(\bar{d}_{i L} \rho^{-}-\bar{u}_{i L} \rho^{0 *}+\bar{D}_{i L} \rho^{--}\right) u_{a R} \\
& +\frac{\lambda_{1 a}^{u}}{\Lambda}\left[\bar{u}_{1 L}\left(\rho^{0 *} \chi^{0 *}-\rho^{--} \chi^{++}\right)+\bar{d}_{1 L}\left(\rho^{--} \chi^{+}-\rho^{-} \chi^{0 *}\right)\right. \\
& \left.+\bar{T}_{L}\left(\rho^{-} \chi^{++}-\rho^{0 *} \chi^{+}\right)\right] u_{a R}+H . c .
\end{aligned}
$$

In the basis $\left(u_{1}, u_{2}, u_{3}\right)$, the up-type quarks mass matrix is given by

$$
m_{u}=\frac{v_{\rho}}{\sqrt{2}}\left(\begin{array}{ccc}
\lambda_{11}^{u} \frac{v_{\chi}}{\sqrt{2} \Lambda} & \lambda_{12}^{u} \frac{v_{\chi}}{\sqrt{2} \Lambda} & \lambda_{13}^{u} \frac{v_{\chi}}{\sqrt{2} \Lambda} \\
-\lambda_{21}^{u} & -\lambda_{22}^{u} & -\lambda_{23}^{u} \\
-\lambda_{31}^{u} & -\lambda_{32}^{u} & -\lambda_{33}^{u}
\end{array}\right)
$$

For down quark sector, the relevant Yukawa interactions are

$$
\begin{aligned}
-L_{Y u k}^{d} & =\lambda_{1 a}^{d} \bar{Q}_{1 L} \rho d_{a R}+\frac{\lambda_{i a}^{d}}{\Lambda} \varepsilon_{n m p}\left(\bar{Q}_{i L n} \rho_{m} \chi_{p}\right) d_{a R}+H . c . \\
& =\lambda_{1 a}^{d}\left(\bar{u}_{1 L} \rho^{+}+\bar{d}_{1 L} \rho^{0}+\bar{T}_{L} \rho^{++}\right) d_{a R} \\
& +\frac{\lambda_{i a}^{d}}{\Lambda}\left[\bar{d}_{i L}\left(\rho^{0} \chi^{0}-\rho^{++} \chi^{--}\right)+\bar{u}_{i L}\left(\rho^{+} \chi^{0}-\rho^{++} \chi^{-}\right)\right. \\
& \left.+\bar{D}_{i L}\left(\rho^{+} \chi^{--}-\rho^{0} \chi^{-}\right)\right] d_{a R}+H . c .
\end{aligned}
$$

Thus, in the basis $\left(d_{1}, d_{2}, d_{3}\right)$, the mass matrix for the down-type quarks is

$$
m_{d}=\frac{v_{\rho}}{\sqrt{2}}\left(\begin{array}{ccc}
\lambda_{11}^{d} & \lambda_{12}^{d} & \lambda_{13}^{d} \\
\lambda_{21}^{d} \frac{v_{\chi}}{\sqrt{2} \Lambda} & \lambda_{22}^{d} \frac{v_{\chi}}{\sqrt{2} \Lambda} & \lambda_{23}^{d} \frac{v_{\chi}}{\sqrt{2} \Lambda} \\
\lambda_{31}^{d} \frac{v_{\chi}}{\sqrt{2} \Lambda} & \lambda_{32}^{d} \frac{v_{\chi}}{\sqrt{2} \Lambda} & \lambda_{33}^{d} \frac{v_{\chi}}{\sqrt{2} \Lambda}
\end{array}\right)
$$

It was shown that as the minimal version, this model is perturbatively reliable at the scale around $\Lambda=4-5 \mathrm{TeV}$ [11]. In the model under consideration, there are 18 free Yukawa couplings to generate masses for 6 quarks only. For a naive analysis [6], we just take the diagonal case where

$$
\begin{aligned}
& m_{u} \approx \lambda_{11}^{u} \frac{v_{\chi} v_{\rho}}{2 \Lambda}, \quad m_{d} \approx \lambda_{11}^{d} \frac{v_{\rho}}{\sqrt{2}}, \quad m_{s} \approx \lambda_{22}^{d} \frac{v_{\chi} v_{\rho}}{2 \Lambda}, \\
& m_{c} \approx-\lambda_{22}^{u} \frac{v_{\rho}}{\sqrt{2}}, \quad m_{b} \approx \lambda_{33}^{d} \frac{v_{\chi} v_{\rho}}{2 \Lambda}, \quad m_{t} \approx-\lambda_{33}^{u} \frac{v_{\rho}}{\sqrt{2}} .
\end{aligned}
$$

For sake of simplicity, assuming $\Lambda=5 \mathrm{TeV}, v_{\chi}=1 \mathrm{TeV}, m_{u}=2.5 \mathrm{MeV}, m_{d}=4.95 \mathrm{MeV}, m_{s}=$ $105 \mathrm{MeV}, m_{c}=1.26 \mathrm{GeV}, m_{b}=4.25 \mathrm{GeV}$ and $m_{t}=173 \mathrm{GeV}$, we get then [6] $\lambda_{11}^{u} \approx 10^{-3}$, $\lambda_{11}^{d} \approx 2.8 \times 10^{-5}, \lambda_{22}^{d} \approx 2.1 \times 10^{-2}, \lambda_{22}^{u} \approx-7.24 \times 10^{-3}, \lambda_{33}^{d} \approx 8.5 \times 10^{-1}, \lambda_{33}^{u} \approx-1.03$.

With a scale $\Lambda \sim 4-5 \mathrm{TeV}$, to guarantee the proton stability, as in Ref. [12], a discrete $Z_{2}$ symmetry over the quark fields

$$
Q_{a L} \rightarrow-Q_{a L}, \quad q_{a R} \rightarrow-q_{a R}
$$

should be imposed. 

tons [6]

The following effective five-dimension operator will generate masses for the charged lep-

$$
\begin{aligned}
L_{Y u k}^{l}= & \frac{\kappa_{l}}{\Lambda}\left(\overline{f_{L}^{c}} \rho^{*}\right)\left(\chi^{\dagger} f_{L}\right)+H . c . \\
= & \frac{\kappa^{l}}{\Lambda}\left[\overline{\nu_{L}^{c}} \rho^{-}+\overline{l_{L}^{c}} \rho^{0 *}+\overline{l_{L}} \rho^{--}\right]\left[\nu_{L} \chi^{+}+l_{L} \chi^{++}+l_{L}^{c} \chi^{0 *}\right]+H . c . \\
= & \frac{\kappa^{l}}{\Lambda}\left[\overline{\nu_{L}^{c}} \rho^{-} \nu_{L} \chi^{+}+\overline{\nu_{L}^{c}} \rho^{-} l_{L}\left(c_{\alpha} \tilde{h}^{++}+s_{\alpha} h^{++}\right)+\frac{1}{\sqrt{2}} \overline{\nu_{L}^{c}} \rho^{-} l_{L}^{c}\left(v_{\chi}-i I_{\chi}\right)\right] \\
& +\frac{\kappa^{l}}{\Lambda \sqrt{2}} \overline{\nu_{L}^{c}} \rho^{-} l_{L}^{c}\left(c_{\beta} h_{2}-s_{\beta} h_{1}\right) \\
& +\frac{\kappa^{l}}{\Lambda \sqrt{2}} \overline{l_{L}^{c}}\left(v_{\rho}-i I_{\rho}\right)\left[\nu_{L} \chi^{+}+l_{L}\left(c_{\alpha} \tilde{h}^{++}+s_{\alpha} h^{++}\right)\right] \\
& +\frac{\kappa^{l}}{2 \Lambda} \overline{l_{L}^{c}}\left(v_{\rho}-i I_{\rho}\right) l_{L}^{c}\left(v_{\chi}-i I_{\chi}\right) \\
& +\frac{\kappa^{l}}{2 \Lambda} \overline{l_{L}^{c}}\left(v_{\rho}-i I_{\rho}\right) l_{L}^{c}\left(c_{\beta} h_{2}-s_{\beta} h_{1}\right) \\
& +\frac{\kappa^{l}}{\Lambda \sqrt{2}} \overline{l_{L}^{c}}\left(c_{\beta} h_{1}+s_{\beta} h_{2}\right)\left[\nu_{L} \chi^{+}+l_{L}\left(c_{\alpha} \tilde{h}^{++}+s_{\alpha} h^{++}\right)\right] \\
& +\frac{\kappa^{l}}{2 \Lambda} \overline{l_{L}^{c}}\left(c_{\beta} h_{1}+s_{\beta} h_{2}\right) l_{L}^{c}\left(v_{\chi}-i I_{\chi}\right)+\frac{\kappa^{l}}{2 \Lambda} \overline{l_{L}^{c}}\left(c_{\beta} h_{1}+s_{\beta} h_{2}\right) l_{L}^{c}\left(c_{\beta} h_{2}-s_{\beta} h_{1}\right) \\
& +\frac{\kappa^{l}}{\Lambda} \overline{l_{L}}\left(c_{\alpha} h^{--}-s_{\alpha} \tilde{h}^{--}\right)\left[\nu_{L} \chi^{+}+l_{L}\left(c_{\alpha} \tilde{h}^{++}+s_{\alpha} h^{++}\right)+\frac{l_{L}^{c}}{\sqrt{2}}\left(v_{\chi}-i I_{\chi}\right)\right] \\
& +\frac{\kappa^{l}}{\sqrt{2} \Lambda} \overline{l_{L}}\left(c_{\alpha} h^{--}-s_{\alpha} \tilde{h}^{--}\right) l_{L}^{c}\left(c_{\beta} h_{2}-s_{\beta} h_{1}\right)+H . c .
\end{aligned}
$$

From (27), it follows masses for the charged leptons $m_{l}=\frac{v_{\chi}}{\Lambda} \kappa_{l} v_{\rho} \approx \kappa_{l} v_{\rho}$. Taking into account $m_{e}=0.5 \mathrm{MeV}, m_{\mu}=105 \mathrm{MeV}, m_{\tau}=1.77 \mathrm{GeV}$, one gets [6] $k_{e}=2 \times 10^{-5}, k_{\mu}=4.3 \times 10^{-3}$ and $k_{\tau}=7.2 \times 10^{-2}$.

From (27), it follows the interaction

$$
\frac{\kappa^{l} c_{\alpha} v_{\chi}}{\sqrt{2} \Lambda} h^{--} \bar{l}_{L} l_{L}^{c} \approx \frac{\kappa^{l} c_{\alpha}}{\sqrt{2}} h^{--} \bar{l}_{L} l_{L}^{c}
$$

which is responsible for lepton-number violating decay of $h^{--}$to two charged leptons. This would be a specific character of the model.

Generation for correct neutrino mass, in this model, is still open question [6].

\section{CHARGED AND NEUTRAL CURRENTS}

The interactions among the gauge bosons and fermions are read off from

$$
\mathcal{L}_{F}=\bar{R} i \gamma^{\mu}\left(\partial_{\mu}-i g_{X} B_{\mu} X\right) R
$$




$$
+\quad \bar{L} i \gamma^{\mu}\left(\partial_{\mu}-i \frac{g_{X}}{\sqrt{6}} B_{\mu} X-i g \sum_{a=1}^{8} W_{\mu}^{a} \cdot \frac{\lambda_{a}}{2}\right) L,
$$

where $R$ represents any right-handed singlet and $L$ any left-handed triplet or antitriplet.

The interactions among the charged vector fields with leptons are [6]

$$
\begin{aligned}
\mathcal{L}_{l}^{C C} & =\frac{g}{\sqrt{2}}\left(\bar{\nu}_{a L} \gamma^{\mu} V_{P M N S}^{l} e_{a L} W_{\mu}^{+}+\bar{e}^{c}{ }_{a R} O^{V} \gamma^{\mu} \nu_{a_{L}} V_{\mu}^{+}\right. \\
& \left.+\bar{e}_{a L} \gamma^{\mu} e_{a R}^{c} U_{\mu}^{++}+\text {H.c. }\right) .
\end{aligned}
$$

with $V_{P M N S}^{l}=V_{L}^{\nu \dagger}$ being the PMNS mixing matrix and $O^{V}=V_{L}^{\nu}$ is the matrix diagonalizing neutrino mass one.

For the quarks we have

$$
\begin{aligned}
\mathcal{L}_{q}^{C C} & =\frac{g}{\sqrt{2}}\left[\bar{u}_{L} V_{C K M}^{q} \gamma^{\mu} d_{L} W_{\mu}^{+}+\left(\bar{T}_{L} \gamma^{\mu}\left(V_{L}^{u}\right)_{1 a} u_{a L}-\bar{d}_{l L} \gamma^{\mu}\left(V_{L}^{d \dagger}\right)_{l i} D_{i_{L}}\right) V_{\mu}^{+}\right. \\
& \left.+\left(\bar{u}_{l_{L}}\left(V_{L}^{u \dagger}\right)_{l i} \gamma^{\mu} D_{i_{L}}+\bar{T}_{L} \gamma^{\mu}\left(V_{L}^{d}\right)_{1 a} d_{a_{L}}\right) U_{\mu}^{++}+\text {H.c. }\right]
\end{aligned}
$$

where $i, l=2,3, V_{C K M}^{q}=V_{L}^{u \dagger} V_{L}^{d}$ is the CKM mixing matrix. One assumes that the exotic quarks come in a diagonal basis.

We can see that the interactions with the $V^{+}$and $U^{++}$bosons violate the lepton number (see Eq.(29)) and the weak isospin (see Eq.(30)).

The electromagnetic current for fermions is the usual one

$$
Q_{f} e \bar{f} \gamma^{\mu} f A_{\mu},
$$

where $f$ is any fermion with $Q_{f}=0,-1,2 / 3,-1 / 3,5 / 3,-4 / 3$ and the electromagnetic coupling constant $e$ is identified as follows

$$
e=g \sin \theta_{W}
$$

The neutral current interactions can be written in the form

$$
\begin{aligned}
\mathcal{L}^{N C} & =\frac{g}{2 c_{W}}\left\{\bar{f} \gamma^{\mu}\left[a_{1 L}(f)\left(1-\gamma_{5}\right)+a_{1 R}(f)\left(1+\gamma_{5}\right)\right] f Z_{\mu}^{1}\right. \\
& \left.+\bar{f} \gamma^{\mu}\left[a_{2 L}(f)\left(1-\gamma_{5}\right)+a_{2 R}(f)\left(1+\gamma_{5}\right)\right] f Z_{\mu}^{2}\right\} .
\end{aligned}
$$

The couplings of fermions with $Z^{1}$ and $Z^{2}$ bosons are given as follows

$$
\begin{aligned}
a_{1 L, R}(f) & =\cos \phi\left[T^{3}\left(f_{L, R}\right)-s_{W}^{2} Q(f)\right] \\
& -\sin \phi\left[\frac{X\left(f_{L, R}\right)}{\sqrt{3}}\left(\frac{1-s_{W}^{2}}{\sqrt{1-4 s_{W}^{2}}}\right)-\frac{\sqrt{1-4 s_{W}^{2}}}{2 \sqrt{3}} Y\left(f_{L, R}\right)\right], \\
a_{2 L, R}(f) & =\cos \phi\left[\frac{X\left(f_{L, R}\right)}{\sqrt{3}}\left(\frac{1-s_{W}^{2}}{\sqrt{1-4 s_{W}^{2}}}\right)-\frac{\sqrt{1-4 s_{W}^{2}}}{2 \sqrt{3}} Y\left(f_{L, R}\right)\right] \\
& +\sin \phi\left[T^{3}\left(f_{L, R}\right)-s_{W}^{2} Q(f)\right],
\end{aligned}
$$

where $T^{3}(f)$ and $Q(f)$ are, respectively, the third component of the weak isospin and the charge of the fermion $f$. Note that for the exotic quarks, the weak isospin is equal to zero. Eqs. (34) 
are valid for both left- and right-handed currents. Since the value of $X$ is different for triplets and antitriplets, the $Z^{2}$ coupling to left-handed ordinary quarks is different for the first family, and thus flavor changing.

We can also express the neutral current interactions of Eq. (33) in terms of the vector and axial-vector couplings as follows

$$
\begin{aligned}
\mathcal{L}^{N C} & =\frac{g}{2 c_{W}}\left\{\bar{f} \gamma^{\mu}\left[g_{1 V}(f)-g_{1 A}(f) \gamma_{5}\right] f Z_{\mu}^{1}\right. \\
& \left.+\bar{f} \gamma^{\mu}\left[g_{2 V}(f)-g_{2 A}(f) \gamma_{5}\right] f Z_{\mu}^{2}\right\} .
\end{aligned}
$$

The values of these couplings are

$$
\begin{aligned}
g_{1 V}(f) & =\cos \phi\left[T^{3}\left(f_{L}\right)-2 s_{W}^{2} Q(f)\right] \\
& -\sin \phi\left[\frac{X\left(f_{L}\right)}{\sqrt{3}}\left(\frac{1-s_{W}^{2}}{\sqrt{1-4 s_{W}^{2}}}\right)-\frac{\sqrt{1-4 s_{W}^{2}}}{2 \sqrt{3}} Y\left(f_{L}\right)+\frac{Q\left(f_{R}\right)}{\sqrt{3}}\left(\frac{3 s_{W}^{2}}{\sqrt{1-4 s_{W}^{2}}}\right)\right], \\
g_{1 A}(f) & =\cos \phi T^{3}\left(f_{L}\right) \\
& -\sin \phi\left[\frac{X\left(f_{L}\right)}{\sqrt{3}}\left(\frac{1-s_{W}^{2}}{\sqrt{1-4 s_{W}^{2}}}\right)-\frac{\sqrt{1-4 s_{W}^{2}}}{2 \sqrt{3}} Y\left(f_{L}\right)-\frac{Q\left(f_{R}\right)}{\sqrt{3}}\left(\frac{3 s_{W}^{2}}{\sqrt{1-4 s_{W}^{2}}}\right)\right]
\end{aligned}
$$

where we have used $Q\left(f_{R}\right)=X\left(f_{R}\right)$ for the singlets. The values of $g_{1 V}, g_{1 A}$ and $g_{2 V}, g_{2 A}$ are listed in Tables 3, where the first generation is assumed to belong to the triplet. However, to get some indication as to why the top quark is so heavy, we have to treat the third generation differently from the first two as in Refs [2] and [13].

Table 3. The $Z^{1} \rightarrow f \bar{f}$ couplings in the RM 331 model.

\begin{tabular}{|c|c|c|}
\hline $\mathrm{f}$ & $g_{1 V}(f)$ & $g_{1 A}(f)$ \\
\hline$e, \mu, \tau$ & $\left(-\frac{1}{2}+2 s_{W}^{2}\right) \cos \phi-\sin \phi \frac{\sqrt{3\left(1-4 s_{W}^{2}\right)}}{2}$ & $-\frac{1}{2} \cos \phi-\sin \phi \frac{\sqrt{\left(1-4 s_{W}^{2}\right)}}{2 \sqrt{3}}$ \\
\hline$\nu_{e}, \nu_{\mu}, \nu_{\tau}$ & $\frac{1}{2}\left(\cos \phi-\sin \phi \frac{\left(1-4 s_{W}^{2}\right)^{1 / 2}}{\sqrt{3}}\right)$ & $\frac{1}{2}\left(\cos \phi-\sin \phi \frac{\left(1-4 s_{W}^{2}\right)^{1 / 2}}{\sqrt{3}}\right)$ \\
\hline $\mathrm{t}$ & $\left(\frac{1}{2}-\frac{4 s_{W}^{2}}{3}\right) \cos \phi-\sin \phi \frac{1+4 s_{W}^{2}}{2 \sqrt{3}\left(1-4 s_{W}^{2}\right)^{1 / 2}}$ & $\frac{1}{2} \cos \phi-\sin \phi \frac{\left(1-4 s_{W}^{2}\right)^{1 / 2}}{2 \sqrt{3}}$ \\
\hline $\mathrm{b}$ & $\left(-\frac{1}{2}+\frac{2 s_{W}^{2}}{3}\right) \cos \phi-\sin \phi \frac{1-2 s_{W}^{2}}{2 \sqrt{3\left(1-4 s_{W}^{2}\right)}}$ & $-\frac{1}{2} \cos \phi-\sin \phi \frac{1+2 s_{W}^{2}}{2 \sqrt{3\left(1-4 s_{W}^{2}\right)}}$ \\
\hline $\mathrm{u}, \mathrm{c}$ & $\left(\frac{1}{2}-\frac{4 s_{W}^{2}}{3}\right) \cos \phi+\sin \phi \frac{1-6 s_{W}^{2}}{2 \sqrt{3\left(1-4 s_{W}^{2}\right)}}$ & $\frac{1}{2} \cos \phi+\sin \phi \frac{1+2 s_{W}^{2}}{2 \sqrt{3\left(1-4 s_{W}^{2}\right)}}$ \\
\hline $\mathrm{d}, \mathrm{s}$ & $\left(-\frac{1}{2}+\frac{2 s_{W}^{2}}{3}\right) \cos \phi+\sin \phi \frac{1}{2 \sqrt{3\left(1-4 s_{W}^{2}\right)}}$ & $-\frac{1}{2} \cos \phi+\sin \phi \frac{1}{2 \sqrt{3}}\left(1-4 s_{W}^{2}\right)^{1 / 2}$ \\
\hline$T$ & $-\frac{10}{3} s_{W}^{2} \cos \phi+\sin \phi \frac{1-11 s_{W}^{2}}{2 \sqrt{3\left(1-4 s_{W}^{2}\right)}}$ & $\sin \phi \frac{1-s_{W}^{2}}{2 \sqrt{3\left(1-4 s_{W}^{2}\right)}}$ \\
\hline$D_{i}$ & $\frac{8}{3} s_{W}^{2} \cos \phi-\sin \phi \frac{1-9 s_{W}^{2}}{2 \sqrt{3\left(1-4 s_{W}^{2}\right)}}$ & $-\sin \phi \frac{1-s_{W}^{2}}{\sqrt{3\left(1-4 s_{W}^{2}\right)}}$ \\
\hline
\end{tabular}


We can realize that in the limit $\phi=0$ the couplings to $Z^{1}$ of the ordinary leptons and quarks are the same as in the SM. Furthermore, the electric charge defined in Eq. (32) agrees with the SM. Because of this, we can test the new phenomenology beyond the SM.

In the model under consideration, the interactions with the heavy charged vector bosons $V^{+}, U^{++}$violate the lepton number and the weak isospin. Because of the mixing, the mass eigenstate $Z^{1}$ now picks up flavor-changing couplings proportional to $\sin \phi$. However, since $Z-Z^{\prime}$ mixing is constrained to be very small, evidence of FCNC's in the 3-3-1 model can only be probed indirectly at present via the $Z^{2}$ couplings.

\section{CONSTRAINTS ON THE $Z-Z^{\prime}$ MIXING ANGLE AND THE $Z^{2}$ MASS}

There are many ways to get constraints on the mixing angle $\phi$ and the $Z^{2}$ mass. Below we present a simple one. A constraint on the $Z-Z^{\prime}$ mixing can be followed from the $Z$ data. Hence we now calculate the $Z$ width in this model.

The decay width of the $Z$ boson is described by [14-16]

$$
\Gamma(f \bar{f})=\frac{\rho G_{F} M_{Z}^{3}}{6 \sqrt{2} \pi} N_{c}^{f}\left(\beta^{2}\left|\bar{g}_{1 A}^{f}\right|^{2}+\frac{3 \beta-\beta^{3}}{2}\left|\bar{g}_{1 V}^{f}\right|^{2}\right)\left(1+n_{f}\right) R_{E W} R_{Q C D},
$$

where $\beta=\sqrt{1-4 \frac{m_{f}^{2}}{M_{Z}^{2}}}[16], \beta$ is very small and we present a result which is correct up to terms of order $\alpha \alpha_{s}$ :

$$
\Gamma(f \bar{f})=\frac{\rho G_{F} M_{Z}^{3}}{6 \sqrt{2} \pi} N_{c}^{f}\left(\left|\bar{g}_{1 A}^{f}\right|^{2} R_{A}^{f}+\left|\bar{g}_{1 V}^{f}\right|^{2} R_{V}^{f}\right)\left(1+n_{f}\right),
$$

where $N_{C}^{f}$ is the color factor and other parameters are given in Ref. [16]

$$
\begin{aligned}
& \rho=1+\delta \rho, \delta \rho_{f \neq b}=\frac{3 G_{F} m_{t}^{2}}{8 \sqrt{2} \pi^{2}}, \\
& \delta \rho_{f=b}=-\frac{G_{F} m_{t}^{2}}{2 \sqrt{2} \pi^{2}}, n_{b}=10^{-2}\left(\frac{1}{5}-\frac{m_{t}^{2}}{2 m_{Z}^{2}}\right), n_{f \neq b} \sim 0 .
\end{aligned}
$$

Here $R_{A}^{f}$ and $R_{V}^{f}$ are radiator factors to account for final state QED and QCD corrections, as well as effects due to nonzero fermions masses.

The non-factorial electroweak correction is given by [15]

$$
R_{V}^{f}=1+\frac{3 \alpha\left(M_{Z}\right)}{4 \pi}, \quad R_{A}^{f}=1-6 \frac{m_{l}^{2}}{M_{Z}^{2}}+\frac{3 \alpha\left(M_{Z}\right)}{4 \pi},
$$

where $\alpha\left(M_{Z}\right)$ denotes the QED coupling constant at the scale $M_{Z}$. The QCD correction is given by

$$
R_{V}^{f}(s)=R_{A}^{f}(s)=1+\frac{3 \alpha_{s}}{4 \pi} Q_{f}^{2}+\frac{\alpha_{s}}{\pi}+\mathcal{O}\left(\alpha^{2}\right) .
$$

By assuming the masses of all the ordinary fermions except the $t$ quark to be much lighter than the mass of the $Z$ boson and the masses of the exotic quarks to be much heavier than the mass of the $Z$ boson, the total width of the $Z$ boson is given as

$$
\begin{aligned}
\Gamma_{\text {total }}^{R M 31}[\mathrm{GeV}] & =2.49632+1.6968 \sin 2 \phi+\mathcal{O}\left(\sin ^{2} \phi\right), \\
\Gamma_{b \bar{b}}^{R M 331}[\mathrm{GeV}] & =0.377046+0.98375 \sin 2 \phi+\mathcal{O}\left(\sin ^{2} \phi\right),
\end{aligned}
$$




$$
\Gamma_{\text {hadrons }}^{R M 331}[\mathrm{GeV}]=1.74022+1.5683 \sin 2 \phi+\mathcal{O}\left(\sin ^{2} \phi\right),
$$

where we have used [1]: $G_{F}=1.166378 .10^{-5} \mathrm{GeV}^{-2}, \alpha^{-1}\left(M_{Z}\right)=128.87, \quad \alpha_{s}=0.1184$, $\bar{s}_{W}^{2}\left(M_{Z}\right)=0.23116, \frac{M_{\tau}}{M_{Z}}=1.7768 / 91.187$ and $m_{t}=173.5 \mathrm{GeV}$.

Taking the experimental result [1]: $\Gamma_{\text {total }}[\mathrm{GeV}]=2.4952 \pm 0.0023$, we obtain the limit for the mixing angle

$$
-0.001 \leq \phi \leq 0.00034 \text {. }
$$

Next, let us consider $R_{b} \equiv \frac{\Gamma(b \bar{b})}{\Gamma(\text { hadrons })}$. In the model under consideration, from (39) and (40), we obtain

$$
R_{b}^{R M 331}=0.21666+0.740083 \tan \phi+\mathcal{O}\left(\tan ^{2} \phi\right) .
$$

According to the experimental result $R_{b}=0.21629 \pm 0.00066$ [1], we also get

$$
-0.001397 \leq \phi \leq 0.00038 \text {. }
$$

Thus, both limits of the mixing angle in (41) and in (43) are consistent: $|\phi| \leq 10^{-3}$. Therefore, hereafter we can set $\phi=0$. For the shorthand, we rename $Z_{1}$ to be $Z$ and $Z_{2}$ to be $Z^{\prime}$.

\section{FLAVOR-CHANGING NEUTRAL CURRENTS AND MASS DIFFERENCE OF THE NEUTRAL MESON SYSTEMS}

Due to the fact that one family of left-handed quarks is treated differently from the other two, the $X$ charges for left-handed quarks are different too (see Eq. (1)). Therefore flavor-changing neutral currents $Z_{1}, Z_{2}$ occur through a mismatch between weak and mass eigenstates.

Let us diagolize mass matrices by three biunitary transformations

$$
\begin{aligned}
U_{L}^{\prime} & =V_{L}^{U} U_{L}, U_{R}^{\prime}=V_{R}^{U} U_{R}, \\
D_{L}^{\prime} & =V_{L}^{D} D_{L}, D_{R}^{\prime}=V_{R}^{D} D_{R},
\end{aligned}
$$

where $U \equiv(u, c, t)^{T}, D \equiv(d, s, b)^{T}$.

The usual Cabibbo-Kobayashi-Maskawa matrix is given by

$$
V_{C K M}=V_{L}^{U+} V_{L}^{D} \text {. }
$$

Using unitarity of the $V^{D}$ and $V^{U}$ matrices, we get flavour-changing neutral interactions

$$
\begin{aligned}
\mathcal{L}_{d s}^{N C} & =\frac{g c_{W}}{2 \sqrt{1-4 s_{W}^{2}}}\left[V_{L i d}^{D *} V_{L i s}^{D}\right] \bar{d}_{L} \gamma_{\mu} s_{L}\left(\cos \phi Z_{2}^{\mu}-\sin \phi Z_{1}^{\mu}\right), \\
\mathcal{L}_{u c}^{N C} & =\frac{g c_{W}}{2 \sqrt{1-4 s_{W}^{2}}}\left[V_{L i u}^{U *} V_{L i c}^{U}\right] \bar{u}_{L} \gamma_{\mu} c_{L}\left(\cos \phi Z_{2}^{\mu}-\sin \phi Z_{1}^{\mu}\right), \\
\mathcal{L}_{d b}^{N C} & =\frac{g c_{W}}{2 \sqrt{1-4 s_{W}^{2}}}\left[V_{L i d}^{D *} V_{L i b}^{D}\right] \bar{d}_{L} \gamma_{\mu} b_{L}\left(\cos \phi Z_{2}^{\mu}-\sin \phi Z_{1}^{\mu}\right),
\end{aligned}
$$

where $i$ denotes the number of "different" quark family i.e. the $\mathrm{SU}(3)_{L}$ quark triplet.

For the neutral kaon system, we get then effective Lagrangian

$$
\mathcal{L}_{\text {eff }}^{\Delta S=2}=\frac{\sqrt{2} G_{F} c_{W}^{4} \cos ^{2} \phi}{\left(1-4 s_{W}^{2}\right)}\left[V_{L i d}^{D *} V_{L i s}^{D}\right]^{2}\left|\bar{d}_{L} \gamma^{\mu} s_{L}\right|^{2}\left(\frac{m_{Z_{1}}^{2}}{M_{Z_{2}}^{2}}+\tan ^{2} \phi\right) .
$$


Similar expressions can be easily written out for $D^{0}-\bar{D}^{0}$ and $B^{0}-\bar{B}^{0}$ systems. From (47) it is straightforward to get the mass difference

$$
\Delta m_{P}=\frac{4 G_{F} c_{W}^{4} \cos ^{2} \phi}{3 \sqrt{2}\left(1-4 s_{W}^{2}\right)}\left[V_{L i d}^{D *} V_{L i \alpha}^{D}\right]^{2}\left(\frac{m_{Z_{1}}^{2}}{M_{Z_{2}}^{2}}+\tan ^{2} \phi\right) f_{P}^{2} B_{P} m_{P},
$$

where $\alpha=s$ for the $K_{L}-K_{S}$ and $\alpha=b$ for the $B^{0}-\bar{B}^{0}$ mixing systems. The $D^{0}-\bar{D}^{0}$ mass difference is given by the expression for the $K^{0}$ system with replace of $V^{D}$ by $V^{U}$. In the previous section, the $Z-Z^{\prime}$ mixing angle $\phi$ was bounded ${ }^{1}:|\phi| \leq 10^{-3}$, hence if $M_{Z_{2}}$ is in order of one hundred $\mathrm{TeV}$, the $Z-Z^{\prime}$ mixing has to be taken into account.

In the usual case, the $Z-Z^{\prime}$ mixing is constrained to be very small, it can be safely neglected. Therefore FCNC's occur only via $Z_{2}$ couplings. For the shothand hereafter we rename $Z_{1}$ to be $Z$ and $Z_{2}$ to be $Z^{\prime}$.

Since it is generally recognized that the most stringent limit from $\Delta m_{K}$, we shall mainly discuss this quantity. We use the experimental values [1]

$$
\Delta m_{K}=(3.489 \pm 0.009) \times 10^{-12} \mathrm{MeV}, \quad m_{K} \simeq 498 \mathrm{MeV}
$$

and

$$
\sqrt{B_{K}} f_{K}=135 \pm 19 \mathrm{MeV} .
$$

Following the idea of Gaillard and Lee [18], it is reasonable to expect that $Z^{\prime}$ exchange contributes a $\Delta m$ no larger than observed values. Substituting (49) and (50) into (48) we get

$$
M_{Z^{\prime}}>2.63 \times 10^{5} \eta_{Z^{\prime}}\left[\operatorname{Re}\left(V_{\text {Lid }}^{D *} V_{\text {Lis }}^{D}\right)^{2}\right]^{1 / 2} \mathrm{GeV} .
$$

where $\eta_{Z^{\prime}} \approx 0.55$ is the leading order QCD corrections [19].

Let us call $\Delta m_{K}^{\min }, \Delta m_{K}^{r h n}$ contributions to $\Delta m$ from the $Z^{\prime}$ in the minimal 331 model and in the model with r.h. neutrinos, respectively. We have then

$$
R \equiv \frac{\Delta m_{K}^{m i n}}{\Delta m_{K}^{r h n}}=\frac{2\left(3-4 s_{W}^{2}\right)}{3\left(1-4 s_{W}^{2}\right)}=19.7
$$

for [1] $s_{W}^{2}=0.2312$. Because of the denominator, the relation is highly sensitive to the value of the Weinberg angle. It is easy to see that a limit for the $Z^{\prime}$ following from $\mathrm{Eq}(51)$ in the model with r.h.neutrinos is approximately 4.4 times smaller than that in the minimal version.

We use the experimental values ${ }^{2}$ [1] presented in Table 4.

Table 4. Experimental data of $K^{0}, D^{0}$ and $B^{0}$ meson.

\begin{tabular}{cccc}
\hline & $K$ & $D$ & $B$ \\
\hline$\Delta m[\mathrm{MeV}]$ & $(3.483 \pm 0.006) \times 10^{-12}$ & $9.478_{-3.291}^{+3.159} \times 10^{-12}$ & $(3.337 \pm 0.033) \times 10^{-10}$ \\
\hline Mass $[\mathrm{MeV}]$ & $497.614 \pm 0.024$ & $1864.86 \pm 0.13$ & $5279.58 \pm 0.17$ \\
\hline$\sqrt{B_{P}} f_{P}[\mathrm{MeV}]$ & $135 \pm 19$ & $200[20]$ & $244 \pm 26$ \\
\hline
\end{tabular}

$1_{\text {see also }}[9,17]$

${ }^{2}$ According to the experimental value in Ref. [1], $\Delta m_{D^{0}}=1.44_{-0.5}^{+0.48} \times 10^{10} \hbar s^{-1}=1.44_{-0.5}^{+0.48} \times 10^{10} \times$ $6.582119 \times 10^{-22}=9.478_{-3.291}^{+3.159} \times 10^{-12} \mathrm{Mev}$. 
Following the idea of Gaillard and Lee [18], it is reasonable to expect that $Z^{\prime}$ exchange contributes a $\Delta m$ no larger than observed values. Substituting Table 4 into (48) we get

- In $K^{0}-\bar{K}^{0}$ system,

$$
\begin{aligned}
M_{Z^{\prime}} & >1.36658 \times 10^{6} \eta_{Z^{\prime}}\left[\operatorname{Re}\left(V_{L 11}^{D *} V_{L 12}^{D}\right)^{2}\right]^{1 / 2} \mathrm{GeV} \\
& =0.751616 \times 10^{6}\left[\operatorname{Re}\left(V_{L 11}^{D *} V_{L 12}^{D}\right)^{2}\right]^{1 / 2} \mathrm{GeV}
\end{aligned}
$$

- In $D^{0}-\bar{D}^{0}$ system,

$$
\begin{aligned}
M_{Z^{\prime}} & >1.60369 \times 10^{6} \eta_{Z^{\prime}}\left[\operatorname{Re}\left(V_{L 11}^{U *} V_{L 12}^{U}\right)^{2}\right]^{1 / 2} \mathrm{GeV} \\
& =0.882028 \times 10^{6}\left[\operatorname{Re}\left(V_{L 11}^{U *} V_{L 12}^{U}\right)^{2}\right]^{1 / 2} \mathrm{GeV}
\end{aligned}
$$

- In $B^{0}-\bar{B}^{0}$ system,

$$
\begin{aligned}
M_{Z^{\prime}} & >4.54763 \times 10^{5} \eta_{Z^{\prime}}\left[\operatorname{Re}\left(V_{L 11}^{D *} V_{L 13}^{D}\right)^{2}\right]^{1 / 2} \mathrm{GeV} \\
& =2.5012 \times 10^{5}\left[\operatorname{Re}\left(V_{L 11}^{D *} V_{L 13}^{D}\right)^{2}\right]^{1 / 2} \mathrm{GeV}
\end{aligned}
$$

where $\eta_{Z^{\prime}} \approx 0.55$ is the QCD corrections [19].

From the present experimental data we cannot get the constraint on $V_{L i j}^{U, D}$. These matrix elements are only constrained by the Cabibbo-Kobayashi-Maskawa matrix. However, it would seem more natural, if Higgs scalars are associated with fermion generations, to have the choice of nondiagonal elements depends on the fields to which the Higgs scalars couple. By this way, the simple Fritzsch [21] scheme gives us

$$
V_{i j}^{D} \approx\left(\frac{m_{i}}{m_{j}}\right)^{1 / 2}, \quad i<j .
$$

In the model under consideration, the first quark family transforms differently. The quark mass eigenstates are $U=(u, c, t)^{T}$ and $D=(d, s, b)^{T}$. In other models, the third family transforms differently so the value of $\Delta m_{P}$ will be differently and the quark mass eigenstates are $U=(t, u, c)^{T}, D=(b, d, s)^{T}$.

Combining (53), and (56) we get the following bounds on $M_{Z^{\prime}}$ :

$$
\begin{aligned}
& M_{Z^{\prime}} \geq 163.194 \mathrm{TeV}, \quad \text { if the first or the second quark family is different (in triplet), } \\
& M_{Z^{\prime}} \geq 4.032 \mathrm{TeV}, \text { if the third quark family is different }
\end{aligned}
$$

It is noted that the limit in (57) is almost consistent with analysis from $B$-physics [22].

From (57) we see that to keep relatively low bounds on $M_{Z^{\prime}}$ the third family should be the one that is different from the other two i.e. is in triplet.

\section{SUMMARY}

In this paper, we have presented the reduced minimal 3-3-1 model (RM 331) with most economical particle content. Some misprints in the original version of the RM 331 model were corrected.

In this work, the limits on the masses of the bilepton gauge bosons and on the mixing angle among the neutral ones were deduced. From the data on branching decay rates of the $Z$ boson, the $Z$ and $Z^{\prime}$ mixing angle $\phi$ lies at $-0.001 \leq \phi \leq 0.0003$. 
We have studied the FCNC's in the RM 331 model arisen from the family discrimination in this model. This gives a reason to conclude that the third family should be treated differently from the first two. In this sense, the $\Delta m_{K}$ gives us the lower bound on $M_{Z^{\prime}}$ as $6.051 \mathrm{TeV}$.

Due to the simplicity of Higgs sector, number of the model's free parameters is strongly reduced and that increases the predicability. However, the price of Higgs simplicity is that there are non-renormalizable effective operators. In addition, a problem on neutrino masses is still an open question. We hope to return to this stuff in the near future.

\section{ACKNOWLEDGMENT}

This research is funded by Vietnam National Foundation for Science and Technology Development (NAFOSTED) under grant number 103.01-2014.51.

\section{REFERENCES}

[1] Particle Data Group: J. Beringer et al., Phys. Rev. D 86 (2012) 010001.

[2] F. Pisano and V. Pleitez, Phys. Rev. D 46 (1992) 410; P. H. Frampton, Phys. Rev. Lett. 69 (1992) 2889; R. Foot, O. F. Hernandez, F. Pisano and V. Pleitez, Phys. Rev. D 47 (1993) 4158.

[3] M. Singer, J. W. F. Valle and J. Schechter, Phys. Rev. D 22 (1980) 738; R. Foot, H. N. Long and Tuan A. Tran, Phys. Rev. D 50 (1994) 34(R) [arXiv:hep-ph/9402243]; J. C. Montero, F. Pisano and V. Pleitez, Phys. Rev. D 47 (1993) 2918; H. N. Long, Phys. Rev. D 54 (1996) 4691; H. N. Long, Phys. Rev. D 53437 (1996); H. N. Long, Mod. Phys. Lett. A 13 (1998) 1865.

[4] W. A. Ponce, Y. Giraldo and L. A. Sanchez, Phys. Rev. D 67 (2003) 075001.

[5] P. V. Dong, H. N. Long, D. T. Nhung and D. V. Soa, Phys. Rev. D 73 (2006) 035004; P. V. Dong, D. T. Huong, Tr. T. Huong and H. N. Long, Phys. Rev. D 74 (2006) 053003; P. V. Dong, H. T. Hung and H. N. Long, Phys. Rev. D 86 (2012) 033002, for a review, see: P. V. Dong and H. N. Long, Adv. High Energy Phys. 2008 (2008) 739492, [arXiv:0804.3239(hep-ph)].

[6] J. G. Ferreira, Jr, P. R. D. Pinheiro, C. A. de S. Pires and P. S. Rodrigues da Silva, Phys. Rev. D 84 (2011) 095019

[7] M. B. Tully and G. C. Joshi, Phys. Rev. D 64 (2001) 0113001

[8] D. Chang and H. N. Long, Phys. Rev. D 73 (2006) 053006

[9] D. Ng, Phys. Rev. D 49 (1994) 4805.

[10] H. N. Long, D. V. Soa, Nucl. Phys. B 601 (2001) 361; D. T. Binh, D .T. Huong, T. T. Huong, H. N. Long, D. V. Soa, J. Phys. G 29 (2003) 1213.

[11] A. G. Dias, R. Martinez, V. Pleitez, Eur. Phys. J. C 39 (2005) 101. See also, A. G. Dias, V. Pleitez, Phys. Rev. D 80 (2009) 056007.

[12] F. Queiroz, C. A. de S. Pires, P. S. Rodrigues da Silva, Phys. Rev. D 82 (2010) 065018.

[13] J. T. Liu, Phys. Rev. D 50, 542 (1994); J. T. Liu and D. Ng, Phys. Rev. D 50 (1994) 548.

[14] P. Langacker and M. Luo, Phys. Rev. D 45 (1992) 278; J. Hewett and T. Rizzo, Phys. Rep. 183, 193 (1989), and references therein.

[15] D. Bardin and G. Passarino, The Standard Model in the Making, Clarendon Press-Oxford (1999).

[16] D. A. Gutierrez, W. A. Ponce and L. A. Sanchez, Eur. Phys. J. C 46 (2006) 497; For more details, see A. Carcamo, R. Martinez, R. and F. Ochoa, Phys. Rev. D 73 (2006) 035007.

[17] H. N. Long and V. T. Van, J. Phys. G 25 (1999) 2319.

[18] M. K. Gaillard and B. W. Lee, Phys. Rev. D 10 (1974) 897.

[19] F. J. Gilman and M. B. Wise, Phys. Rev. D 27 (1983) 1128.

[20] D. G. Dumm, F. Pisano, and V. Pleitez, Mod. Phys. Lett. A 9 (1994) 1609; F. Pisano, J. A. Silva-Sobrinho and M. D. Tonasse, Phys. Rev. D 58 (1998) 057703.

[21] H. Fritzsch, Phys. Lett. B 73 (1978) 317; Nucl. Phys. B 155 (1979) 189.

[22] A. Buras, F. De Fazio, J. Girrbach, and M. Callucci, JHEP 1302 (2013) 023, arXiv:1211.1237 [hep-ph]. 